
\title{
R Research Square \\ Is There an Advantage with Measured Resection for Femoral Rotation Resection in TKA? A Randomized Controlled Trial
}

\section{Ye Zhang}

affiliated hospital of xuzhou medical university

Jian-Ning Sun

affiliated hospital of xuzhou medical university

Lun An

Xuzhou Medical University

Yu Zhang

affiliated hospital of xuzhou medical university

Xiang-Yang Chen ( $\nabla$ xzchenxiangyang@163.com )

Affiliated Hospital of Xuzhou Medical University https://orcid.org/0000-0001-7376-6179

Shuo Feng ( $\nabla$ xzfs0561@163.com)

affiliated hospital of xuzhou medical university

Research article

Keywords: Total knee arthroplasty, gap balance, gait analysis, eLIBRA sensor

Posted Date: December 19th, 2019

DOl: https://doi.org/10.21203/rs.2.19202/v1

License: (c) (1) This work is licensed under a Creative Commons Attribution 4.0 International License.

Read Full License 


\section{Abstract}

\section{Background}

Total knee arthroplasty (TKA) is performed by gap balance (GB) and measured resection (MR) techniques for femoral rotation resection to achieve knee flexion balance and knee stability. It is still controversial to choose which technique is more advantageous. The purpose of this study was to compare the early clinical efficacy of GB and MR in TKA.

\section{Methods}

This study was a prospective randomized, double-blind controlled trial. From March 2018 to March 2019, 99 patients (99 knees) who underwent TKA at our institution were randomly treated with GB and MR. The cutting thickness of posterior condyle, radiographic findings, medial and lateral compartment pressure, gait analysis, and patients satisfaction were recorded.

\section{Results}

A total of 110 patients were enrolled for unilateral THA. Finally, 99 patients were included for analysis and comparison at 12 months follow-up. In GB group, the angle of Cutting Block to PCA was significantly lower than in the MR group $(P<0.001)$ and the cutting thickness of the medial and lateral condyle was significantly higher than in the MR group $(P<0.05)$. Medial compartment pressure was significantly higher than lateral compartment at $90^{\circ}$ flexion and full flexion in MR $(P<0.05)$. In GB group, Sagittal max knee flexion range and transversal knee rotation range was significantly was significantly larger than that in the MR group $(P<0.05)$. In level of satisfaction at the most recent follow-up, there was no significant difference between the two groups ( $p>0.05)$.

\section{Conclusion}

In TKA, GB or MR techniques for femoral external resection has little effect on daily walking. However, the GB technique has advantages in early postoperative knee stability and knee flexion function improvement.

\section{Introduction}

Total knee arthroplasty (TKA) is one of the effective methods to treat middle-stage and end-stage knee arthritis[1]. According to reports, more than 600,000 people in the United States undergo TKA surgery each year. However, the study shows that $19 \%$ of patients are not satisfied with the outcome of TKA[2]. In TKA, the precise placement of the prosthesis position has a great impact on the clinical effect[3,4]. Therefore, it is a key step to obtain a symmetrically flexion and extension gap to match the prosthesis in the success of TKA. Certainly, the femoral extorsion resection directly affects the flexion gap. Appropriate rotation resection can obtain a good flexion gap, which is beneficial to the functional recovery and obtain 
good contact pressure and patellofemoral joint motion[5, 6]. Asymmetric gaps and incorrect soft tissue balance can lead to instability of knee, patellofemoral joint dysfunction, and anterior knee pain[7].

Gap balance (GB) resection and measurement resection (MR) are two techniques of femoral extorsion resection in TKA surgery[8, 9]. MR technique was performed by the positioning of the Transepicondylar Axes (TEA), Anterior posterior (AP), and Posterior Condylar Axes (PCA) to obtain the flexion gap[3, 10-12]. In the surgical operation, however, it is difficult for the surgeon to accurately identify the bone markers. Yau[13] found that $56 \%, 72 \%$ and $60 \%$ of femoral rotation exceeded the normal range when resection was performed using the TEA, PCA and AP, respectively. GB technique was proposed by Freeman and Install[14-16]. Based on the balance of soft tissue and ligament tension, the distal femoral flexion and extension resection are parallel to the tibial proximal platform. But GB technique may cause internal rotation of a part of the patients' prosthesis and poor patellofemoral joint motion. Therefore, there is still controversy about which surgical technique is used to achieve optimal gap balance.

Whether GB or MR technique, the primary goal is to ensure the stability of the knee prosthesis and good function. Previous studies have compared the two techniques in terms of knee stability, radiographic findings and patient satisfaction[17-19], but the results are still controversial. Moreover, most studies only use subjective indexes such as patient satisfaction, pain score or functional score, and cannot fully evaluate the knee joint performance in daily life. Few studies have used objective gait analysis (Vicon MX) to compare postoperative knee function in two techniques[20,21]. Therefore, we designed a prospective, double-blind, randomized controlled study comparing these two techniques. Through postoperative gait analysis, intraoperative resection and pressure index, postoperative radiographic findings, and subjective satisfaction of patients, the clinical effect was evaluated in two techniques. We hypothesized that GB technique has advantages in early postoperative knee stability and knee flexion function improvement.

\section{2.methods}

2.1 Study design and participants This study was a prospective randomized, double-blind controlled trial and was organized by Department of Orthopedic Surgery in our institution from March 2018 to March 2019. This study was in compliance with the medical institution regulations and conforms to the ethical standards of the national research council and the Helsinki declaration of 1964. This study was approved by our institutional review board (XYFY2018-JS004-01). Informed consent was obtained from all patients. CONSORT Flow Diagram is provided in Fig 1.

Inclusion criteria: (1) The patient was diagnosed osteoarthritis of the knee, with poor conservative treatment effect for 6 months. Suitable for TKA, (2) primary unilateral TKA.

Exclusion criteria: (1) Revision total knee arthroplasty, (2) Knee valgus deformity (3) Severe knee varus deformity $>15^{\circ}$ or bone defect, (4) Osteoarthritis involving other joints of the lower extremities and affecting patient functional activity, (5) Knee joint infection or suspected infection, (6) Patients with 
traumatic arthritis. In addition, patients with a history of cardiac disease, deep vein thrombosis, or surgery of the knee joint were excluded.

\subsection{Randomization and masking}

Basing on the above selection and exclusion criteria, we prospectively investigated the clinical data of 110 patients who received primary TKA from March 2018 to March 2019. Krishna[20, 22] et al. assessed the clinical effect following GB and MR technique for TKA. Based on their results and considering the potential loss to follow-up of $10 \%$, a total of 110 patients were needed. A computerized randomization list was created by a statistician in a random order using a concealed block size of four. The list was used to randomly assign knees to either the GB or MR group. Allocations were sealed in consecutively numbered opaque envelopes. Once the patient agrees to be included in the trial, the next sealed envelope is opened by an independent investigator to randomly assign a resection technique. Patients and clinical data collector were blinded to the group assignment. The data center based at the Department of Orthopedic Surgery was responsible for treatment allocation, central monitoring, and statistical analyses.

\subsection{Gait analysis}

The British 10-Camera Vicon 3D Motion Capture System was used to analyze the postoperative gait of patients (fig 2). Then Polygon Software was used to analyze the temporospatial and kinematics parameters of the gait cycle. Patients wore tights and marked the lower body with a reflective markers. By Capturing the position of the reflective markers at static state, calculation of the alignment of the knee in Sagittal, Coronal and transversal plane was optimized. Patients should walk barefoot on platform at a self-determined walking speed during 10 captured dynamic trials. Kinematic data was collected by Capture System. Our primary goals were temporospatial and kinematic parameters of the sagittal, conormal and transversal plane. degrees of knee joint movement during swing phase were calculated in the three planes. Knee rotation angle was defined between the plane formed by the femoral reflective markers and the plane formed by the tibia reflective markers in transversal plane (Fig 2). Direction of rotation (positive internal vs. negative external rotation) depended on the position of the moving element compared to the fixed element. At the end of the trials, Polygon Software was used to analyze the temporospatial and kinematics parameters of the gait cycle.

\subsection{Procedures}

All patients received general anesthesia in the supine position. Pneumatic tourniquets were used in the surgery. Routine disinfection was performed and towels were applied. Intraoperative femoral extorsion resection was performed by GP and MR techniques, and the distal femur and proximal tibia resection were performed using the same technique. Both groups used the same prosthesis (Zimmer, Posterior stabilized Prosthesis, PS). The incision was closed and drainage was placed When the knee was bent at $45^{\circ}$. The surgical procedures were performed by the Senior surgeon (X.Y.C). Before this study, Dr. C had independently completed hundreds of TKA with GP and MR techniques, and had extensive clinical experience. 
MR: After the distal femur and proximal tibia resection, the knee joint was flexed 90 degrees and femoral external rotation positioning system was selected for external rotation resection. Use the femoral size measurer to determine the femoral prosthesis model, refer to the Posterior Condylar axis (PCA), and select the external rotation angle (usually $3^{\circ}$ external rotation) to determine the osteotomy surface. The femoral size measuring device was used to determine the femoral prosthesis size, and the external rotation angle ( $3^{\circ}$ external rotation) was selected according to PCA to determine the cut thickness. We used the 4-in-1 cutting block localization to perform osteotomy of the anterior condyle, posterior condyle, and intercondylar fossa. A spacer block was placed into the extension gap to confirm it was large enough to accommodate the prosthesis. If the extension gap is unbalanced, the AP and TEA will be used to adjust the external rotation angle for resection or soft tissue release to achieve gap balance.

GB: After completing the distal femur and proximal tibia resection, the assistant lifts the knee to maintain a flexion of $90^{\circ}$ and naturally drooped. The medial and lateral compartments were opened with a lamina spreader, and the femoral posterior condyle resection line was marked according to the knee extension gap, parallel to the tibial osteotomy platform. The 4-in-1 cutting block localization was placed according to the marked line, and the femoral resection was performed after confirmation. We evaluated the extension and flexion gaps using a rectangular module and a pressure sensor. When the pressure difference between the medial and lateral gaps exceeds 15 pounds, soft tissue release is performed.

Intraoperative resection measurement: the angle of cutting block to PCA was measured. After the external rotation resection was completed, the cutting thickness of the medial and lateral condyle (CTMC, CTLC) was measured with Vernier Caliper. Measurements were recorded by independent researcher.

Medial and lateral pressure measurement: pressure measurement was performed using a pressure senor (eLIBRA Dynamic Knee Balancing System, Zimmer) after placing the distal femoral prosthesis mold. After patella reduction, full extension, $45^{\circ}, 90^{\circ}$ and full flexion of the knee joint during passive movement were recorded. The measurements were recorded and converted into pounds ( 1 unit $=3.37$ pounds) by an independent researcher.

Radiographic evaluation: Record the following indexखpreoperative mechanical femorotibial Angle (PremFTA), Post preoperative mechanical femorotibial Angle (Post-mFTA), joint line changes and femoral component rotation Angle (FCRA). FCRA was defined between surgical TEA and posterior condylar line of prosthesis. Measurements were recorded by independent researcher.

\subsection{Post-operative treatment}

All patients used the same nutritional interventions, pain management, antibiotics, thromboprophylaxis, and the same care and rehabilitation exercise regimen. The quadriceps isometric contraction and knee flexion exercise were performed after surgery. The drainage tube was removed 24 hours after surgery. Regular dressing changes are performed by the same surgeon (Y.Z). After extubating, the patients were instructed to exercise with the Continues Passive Motion (CPM) machine, and the patients were informed of the relevant precautions after surgery. All patients underwent subcutaneous injection of low molecular 
weight heparin $(4100 \mathrm{IU} / \mathrm{d})$ on postoperative first day in accordance with the Guidelines for Prevention of Venous Thromboembolism in Major Orthopedic Surgery. Oral rivaroxaban ( $5 \mathrm{mg} / \mathrm{d})$ was continued after discharge to prevent deep vein thrombosis of the lower extremities. The shortest time for anticoagulation is five weeks. Postoperatively, all patients received oral drugs (etoxib, $60 \mathrm{mg} / \mathrm{d}$ ) and ice compress (2 times/d). The suture was removed about 14 days after surgery. X-ray examination of the knee was performed after surgery. The patients underwent routine color Doppler ultrasonography of the lower extremities on postoperative 7th day. X-ray examination of lower extremity and Western Ontario and McMaster University Osteoarthritis Index (WOMAC) was performed before and after surgery. CT examination was performed on postoperative 3rd day. Gait analysis was performed on postoperative 12 months.

\subsection{Statistical analysis}

Data were processed with SPSS 23.0 statistical software and expressed as mean values \pm standard deviation $(x \pm s)$. The categorical variables were compared with a chi-square or Fisher's exact tests, and continuous variables were compared with a Student's t-tests. Data among groups were compared with paired $t$ tests. The test level a value is 0.05 on both sides. The difference was statistically significant at $P$ $<0.05$.

\section{Results}

A total of 110 patients (110 TKAs) enrolled in the study received unilateral THA, and eligible patients will be included in the analysis. Three patients withdrew from the study. Three patients had incorrect resection technique. The data of five patients were lost. This condition resulted in 99 knees in 99 patients for analysis at 12 months follow-up (Fig 1). The surgeon (X.Y.C) completed all operations. There were 19 males and 31 females aged $55.9 \pm 16$.5years on GB group. 19 males and 30 females aged $54.2 \pm 18.7$ on MR group. There was no significant difference between the above data and other baseline data $(P>0.05$, Table 1). Due to the complexity of gait analysis procedures and patients' own willingness, only 83 cases of gait data could be used for analysis (GB: 39 cases, MR: 44 cases, Table 3). Three patients developed superficial infection at the incision and recovered after regular dressing change. The remaining patients incision healed well.

In GB group, the angle of Cutting Block to PCA was statistically lower than in the MR group $(1.5 \pm 2.6 \mathrm{~mm}$ compared with $3.1 \pm 0.5 \mathrm{~mm} \otimes P<0.001)$. And CTMC and CTLC was higher than in the MR group $(9.8 \pm 2.0$ compared with $8.5 \pm 1.2 ; 7.9 \pm 1.8$ compared with $6.8 \pm 1.4, P=0.001, P=0.002$, Table 1, Fig 3).

In pressure measurement, medial compartment pressure was significantly higher than lateral compartment at $90^{\circ}$ flexion and full flexion in MR group (10.9 \pm 7.1 compared with $7.7 \pm 5.3,9.0 \pm 5.2$ compared with $7.1 \pm 4.7, \mathrm{P}<0.05$, Table 2 ). In GB group, there was no statistically significant difference in the medial and lateral pressure. In the process of passive flexion, the medial and lateral compartment pressure decreased with the increase of the degree of flexion. The pressure difference in GB group decreased gradually, while the pressure difference in MR group did not change much (Fig 4). 
In gait analysis, Sagittal max knee flexion range was significantly different between the two groups (52.77 \pm 5.40 compared with $49.52 \pm 7.83, P<0.05$, table 3 ). In the GB group, transversal knee rotation range was significantly larger than that in the MR group ( $3.60 \pm 2.51$ compared with $2.47 \pm 2.52, P<0.05$, Fig 5 ).

\section{4.discussion}

In posterior condyle resection, the cutting thickness in GB group was significantly higher than that in MR group, which obtained a rectangular gap. Therefore, in the gait analysis, the Sagittal knee flexion and the Transversal knee rotation were significantly higher than those of the MR group. Krishna's study[22] are similar to ours. However, some studies have shown that the thickness of posterior condyle in GB is lower than MR[23]. In addition, the cutting thickness is related to the soft tissue release. The cutting thickness of MR is less than GB, and soft tissue release is needed in the later stage. However, less soft tissue release leads to less trauma, which not only helps to reduce postoperative pain, but also contributes to rapid recovery of knee function and improves patients satisfaction [24-26]. Compared with GB technique, in MR we routinely selected anatomical markers as references, and paid attention to the restoration of anatomical morphology. However, in actual operation, it is difficult to locate the bone markers[7, 10]. Kinzel [27] used TEA to control rotation in 74 cases of knee surgery. They found only $75 \%$ of knees would have been within 3 degrees of the true TEA, and the error range was large (range $-6^{\circ}$ to $11^{\circ}$ ). Patients with posterior condyle dysplasia, posterior condyle wear and intra-articular deformities may result in inaccurate locating of bone markers. In contrast, GB technique does not rely on anatomical markers, but the rectangular gap is obtained by tension or self-gravity.

In the pressure measurement, it can be found that the medial pressure between two groups is significantly higher than the lateral pressure in Fig 3. And the pressure has a decreasing trend as the flexion range increased. Previous studies have shown that the knee joint is nearly stable when the pressure difference of medial and lateral compartment is no more than 15 pounds[28, 29]. In this study, the difference was basically within 6 pounds. The conclusions of Kyn et al. are similar to this study[22, 30]. Then Tokuhara[31] et al. measured the medial and lateral gaps of the normal knee by MRI technique and it was found that gaps difference was as high as $4.6 \mathrm{~mm}$ when the flexion was $90^{\circ}$. This also indirectly reflects the pressure difference of the medial and lateral compartments. In addition, Nagai [32] measured the medial and lateral gaps under different tension conditions in GB resection. As the tension and flexion range increased, the medial and lateral gaps also increased but the gap difference did not change much. The rectangular gap obtained and soft tissue balance by GB are better than MR.

Previous studies of gait analysis showed that walking speed and step length of patients with knee osteoarthritis are significantly reduced compared with normal people, and the single support time in the whole gait cycle is significantly longer. The above parameters of patients after TKA will be improved compared with the preoperative parameters, but there is still a certain gap compared with normal gait[3335]. This study mainly compared the effects of GB and MR techniques on postoperative gait in TKA. Only sagittal max knee flexion and transversal knee rotation were significantly different between the two techniques (Table 4). Maier's gait analysis of patients after TKA showed a decrease in sagittal max 
flexion during the swing phase. This conclusion is similar to this study[36]. Huang [37]evaluated the effects of two techniques on the kneeling function after TKA. It showed that GB technique has certain advantages in improving kneeling function, joint mobility and functional improvement after 2 years. One reason may be that the Cut Thickness of posterior condyle in GB was significantly higher than that in MR, which could form a good rectangular flexion gap. So, the movement is closer to normal during flexion.

Our study had several limitations. First, this study only compared the two groups and did not compare with the contralateral knee or the normal knee. In addition, due to the complexity of the gait analysis process and the lack of time for postoperative patients, only 83 patients were available for analysis. Those patients cannot serve as a random sample representative for the entire population of patients, but there is no doubt that this study is also valuable. Second, range of motion was additionally limited by patients pain which can lead to reduced or evading movements. These can affect the collection and analysis of data. Third, the follow-up time of this study was only 12 months. It took longer time to follow up the functional evaluation of the knee. Four, the majority of patients recruited in this study may be different from other regions in knee anatomy $[3,6]$. The demographic characteristics should be noted before extrapolating to other populations.

\section{5.conclusion}

In TKA, the use of GB or MR techniques for femoral external resection has little effect on daily walking, but the GB technique has advantages in early postoperative knee stability and knee flexion function improvement.

\section{Abbreviations}

TKA: Total knee arthroplasty, GB: gap balance, MR: measured resection, BMI: Body Mass Index, ASA: American Society of Anesthesiologists, Pre-mFTA: preoperative mechanical femorotibial angle; PostmFTA, postoperative mechanical femorotibial angle; FCRA, femoral component rotation angle; CTMC, Cut Thickness of Medial Condyle; CTLC, Cut Thickness of Lateral Condyle.; PCA, Posterior Condylar axis. WOMAC: McMaster University Osteoarthritis Index.

\section{Declarations}

\section{Ethics approval and consent to participate}

This study has been approved by the Ethic Committee of the Affiliated Hospital of Xuzhou Medical University (XYFY2018-JS004-01). All patients received informed consent and signed the informed consent.

\section{Consent for publication}

Not applicable. 
Availability of data and materials

All data generated or analyzed during this study are included in this published article, and the supplementary file. We do not wish to share our patients' data because it involves patient's privacy.

\section{Competing interests}

The authors declare that they have no competing interests.

\section{Funding}

The authors received no financial support for the research, authorship, and publication of this article.

\section{Authors' contributions}

YeZ, SF did the study, analyzed the data, and wrote the manuscript. YuZ, JNS, LA,XYC, GCZ was involved in the design, data management, and analysis of the study. All authors read and approved the final manuscript.

\section{Acknowledgements}

The authors thank Rui-Qi Li from Beijing Chao-Yang Hospital affiliated to Capital Medical University for valuable technical assistance and support.

\section{References}

1. Ethgen $\mathrm{O}$, Bruyere $\mathrm{O}$, Richy F, Dardennes C, Reginster JY: Health-related quality of life in total hip and total knee arthroplasty. A qualitative and systematic review of the literature. J Bone Joint Surg Am 2004, 86(5):963-974.

2. Bourne RB, Chesworth BM, Davis AM, Mahomed NN, Charron KDJ: Patient Satisfaction after Total Knee Arthroplasty: Who is Satisfied and Who is Not? Clinical Orthopaedics \& Related Research 2010, 468(1):57-63.

3. Berger RA, Rubash HE, Seel MJ, Thompson WH, Crossett LS: Determining the rotational alignment of the femoral component in total knee arthroplasty using the epicondylar axis. Clin Orthop Relat Res 1993(286):40-47.

4. Luyckx T, Peeters $\mathrm{T}$, Vandenneucker $\mathrm{H}$, Victor $\mathrm{J}$, Bellemans $\mathrm{J}$ : Is adapted measured resection superior to gap-balancing in determining femoral component rotation in total knee replacement? J Bone Joint Surg Br 2012, 94(9):1271-1276.

5. Nikolaides AP, Kenanidis El, Papavasiliou KA, Sayegh FE, Tsitouridis I, Kapetanos GA: Measured resection versus gap balancing technique for femoral rotational alignment: a prospective study. $J$ Orthop Surg (Hong Kong) 2014, 22(2):158-162. 
6. Yip DK, Zhu YH, Chiu KY, Ng TP: Distal rotational alignment of the Chinese femur and its relevance in total knee arthroplasty. J Arthroplasty 2004, 19(5):613-619.

7. Fehring TK: Rotational malalignment of the femoral component in total knee arthroplasty. Clin Orthop Relat Res 2000(380):72-79.

8. Hernandez-Hermoso JA, Nescolarde-Selva L, Rodriguez-Montserrat D, Martinez-Pastor JC, GarciaOltra E, Lopez-Marne S: Different femoral rotation with navigated flexion-gap balanced or measured resection in total knee arthroplasty does not lead to different clinical outcomes. Knee Surg Sports Traumatol Arthrosc 2019.

9. Foge DA, Baldini TH, Hellwinkel JE, Hogan CA, Dayton MR: The Role of Complete Posterior Cruciate Ligament Release in Flexion Gap Balancing for Total Knee Arthroplasty. J Arthroplasty 2019, 34(7s):S361-s365.

10. Sabbioni G, Rani N, Del Piccolo N, Ben Ayad R, Carubbi C, Tigani D: Gap balancing versus measured resection technique using a mobile-bearing prosthesis in computer-assisted surgery. Musculoskelet Surg 2011, 95(1):25-30.

11. Daines BK, Dennis DA: Gap balancing vs. measured resection technique in total knee arthroplasty. Clin Orthop Surg 2014, 6(1):1-8.

12. Kahlenberg CA, Elmasry S, Mayman DJ, Cross MB, Wright TM, Westrich GH, Sculco PK: Posterior condylar bone resection and femoral implant thickness vary by up to $3 \mathrm{~mm}$ across implant systems: implications for flexion gap balancing. Knee Surg Sports Traumatol Arthrosc 2019, 27(7):2140-2144.

13. Yau WP, Chiu KY, Tang WM: How precise is the determination of rotational alignment of the femoral prosthesis in total knee arthroplasty: an in vivo study. J Arthroplasty 2007, 22(7):1042-1048.

14. Freeman MAR, Samuelson KM, Levack B, Alencar PGCD: Knee Arthroplasty at the London Hospital. 1986, \&NA;(205):12.

15. Insall JN, Binazzi R, Soudry M, Mestriner LA: Total knee arthroplasty. 1985, 192(192):13.

16. Elmasry SS, Imhauser CW, Wright TM, Pearle AD, Cross MB, Mayman DJ, Westrich GH, Sculco PK: Neither Anterior nor Posterior Referencing Consistently Balances the Flexion Gap in Measured Resection Total Knee Arthroplasty: A Computational Analysis. J Arthroplasty 2019, 34(5):981986.e981.

17. Churchill JL, Khlopas A, Sultan AA, Harwin SF, Mont MA: Gap-Balancing versus Measured Resection Technique in Total Knee Arthroplasty: A Comparison Study. J Knee Surg 2018, 31(1):13-16.

18. Nagai K, Muratsu H, Takeoka Y, Tsubosaka M, Kuroda R, Matsumoto T: The Influence of Joint Distraction Force on the Soft-Tissue Balance Using Modified Gap-Balancing Technique in PosteriorStabilized Total Knee Arthroplasty. J Arthroplasty 2017, 32(10):2995-2999.

19. Kim CW, Lee CR, Gwak HC, Kim JH, Kwon YU, Kim DY: The Effects of Surgical Technique in Total Knee Arthroplasty for Varus Osteoarthritic Knee on the Rotational Alignment of Femoral Component: Gap Balancing Technique versus Measured Resection Technique. J Knee Surg 2019.

20. Papagiannis GI, Triantafyllou Al, Roumpelakis IM, Papagelopoulos PJ, Babis GC: Gait analysis methodology for the measurement of biomechanical parameters in total knee arthroplasties. A 
literature review. J Orthop 2018, 15(1):181-185.

21. Xu H, Hunt M, Bo Foreman K, Zhao J, Merryweather A: Gait alterations on irregular surface in people with Parkinson's disease. Clin Biomech (Bristol, Avon) 2018, 57:93-98.

22. Cidambi KR, Robertson N, Borges C, Nassif NA, Barnett SL: Intraoperative Comparison of Measured Resection and Gap Balancing Using a Force Sensor: A Prospective, Randomized Controlled Trial. J Arthroplasty 2018, 33(7s):S126-s130.

23. Sun ZH LJ, Tian ZW: Mid囚term follow $\$ up of gap balancing technique in total knee arthroplasty. Orthopedic Journal of China, 21(13):1292-1296.

24. Lee HJ, Lee JS, Jung HJ, Song KS, Yang JJ, Park CW: Comparison of joint line position changes after primary bilateral total knee arthroplasty performed using the navigation-assisted measured gap resection or gap balancing techniques. Knee Surg Sports Traumatol Arthrosc 2011, 19(12):20272032.

25. Moon YW, Kim HJ, Ahn HS, Park CD, Lee DH: Comparison of soft tissue balancing, femoral component rotation, and joint line change between the gap balancing and measured resection techniques in primary total knee arthroplasty: A meta-analysis. Medicine (Baltimore) 2016, 95(39):e5006.

26. Babazadeh S, Dowsey MM, Vasimalla MG, Stoney JD, Choong PFM: Gap Balancing Sacrifices JointLine Maintenance to Improve Gap Symmetry: 5-Year Follow-Up of a Randomized Controlled Trial. J Arthroplasty 2018, 33(1):75-78.

27. Kinzel V, Ledger M, Shakespeare D: Can the epicondylar axis be defined accurately in total knee arthroplasty? Knee 2005, 12(4):293-296.

28. Gustke KA, Golladay GJ, Roche MW, Elson LC, Anderson CR: A new method for defining balance: promising short-term clinical outcomes of sensor-guided TKA. J Arthroplasty 2014, 29(5):955-960.

29. Walker PS, Meere PA, Bell CP: Effects of surgical variables in balancing of total knee replacements using an instrumented tibial trial. Knee 2014, 21(1):156-161.

30. Cho KJ, Seon JK, Jang WY, Park CG, Song EK: Objective quantification of ligament balancing using VERASENSE in measured resection and modified gap balance total knee arthroplasty. BMC Musculoskelet Disord 2018, 19(1):266.

31. Tokuhara Y, Kadoya Y, Nakagawa S, Kobayashi A, Takaoka K: The flexion gap in normal knees. An MRI study. J Bone Joint Surg Br2004, 86(8):1133-1136.

32. Nagai K, Muratsu H, Kanda Y, Tsubosaka M, Kamenaga T, Miya H, Matsushita T, Niikura T, Kuroda R, Matsumoto T: Intraoperative soft tissue balance using novel medial preserving gap technique in posterior-stabilized total knee arthroplasty: comparison to measured resection technique. Knee Surg Sports Traumatol Arthrosc 2018, 26(11):3474-3481.

33. Oberg T, Karsznia A, Oberg K: Basic gait parameters: reference data for normal subjects, 10-79 years of age. J Rehabil Res Dev 1993, 30(2):210-223.

34. Sparkes V, Whatling GM, Biggs P, Khatib N, Al-Amri M, Williams D, Hemming R, Hagen M, Saleem I, Swaminathan $\mathrm{R}$ et al: Comparison of gait, functional activities, and patient-reported outcome 
measures in patients with knee osteoarthritis and healthy adults using 3D motion analysis and activity monitoring: an exploratory case-control analysis. Orthop Res Rev 2019, 11:129-140.

35. Brostrom EW, Esbjornsson AC, von Heideken J, Iversen MD: Gait deviations in individuals with inflammatory joint diseases and osteoarthritis and the usage of three-dimensional gait analysis. Best Pract Res Clin Rheumatol 2012, 26(3):409-422.

36. Maier MW, Aschauer S, Wolf SI, Dreher T, Merle C, Bitsch RG: Three dimensional gait analysis in patients with symptomatic component mal-rotation after total knee arthroplasty. Int Orthop 2019, 43(6):1371-1378.

37. Yi-Fan H, Guo-Dong Z, Guo-Hua W, Lu D, Xin Q: Comparison between different osteotomy methods for kneeling ability recovery after total knee arthroplasty. Chinese Journal of Orthopaedics 2017, 37(11):670-675.

\section{Tables}

Table 1 Baseline patient demographics, intraoperative and radiologic outcome. 


\begin{tabular}{|c|c|c|c|}
\hline & GB & MR & $\mathrm{t}$ or $\mathrm{c} \square \mathrm{P}$ Value \\
\hline \multicolumn{4}{|l|}{ Gender } \\
\hline Male/Female & $19 / 31$ & $19 / 30$ & $0.006 \quad 0.937$ \\
\hline Age (Years) & $55.9 \pm 16.5$ & $54.2 \pm 18.7$ & $0.484 \quad 0.630$ \\
\hline BMI $(\mathrm{kg} / \mathrm{m} \square)$ & $24.3 \pm 2.7$ & $24.2 \pm 3.2$ & $0.074 \quad 0.941$ \\
\hline Diabetes & $4(8 \%)$ & $5(10.2 \%)$ & $0.145 \quad 0.703$ \\
\hline Renal insufficiency & $1(2.0 \%)$ & $2(4.1 \%)$ & $0.365 \quad 0.546$ \\
\hline Smoking & $7(14.0 \%)$ & $6(12.2 \%)$ & 0.0670 .796 \\
\hline ASA & II 40/III 10 & II43/III6 & 1.0980 .295 \\
\hline Immune dysfunction & 0 & 0 & - \\
\hline CTMC (mm) & $9.8 \pm 2.0$ & $8.5 \pm 1.2$ & $3.544 \quad 0.001$ \\
\hline CTLC (mm) & $7.9 \pm 1.8$ & $6.8 \pm 1.4$ & 3.2220 .002 \\
\hline Cutting Block to PCA & $1.7 \pm 1.5$ & $3.1 \pm 0.5$ & $-8.406 \quad 0.000$ \\
\hline Joint Line Change $(\mathrm{mm})$ & $2.5 \pm 3.1$ & $2.0 \pm 2.7$ & 0.8620 .391 \\
\hline Pre-mFTA $\left({ }^{\circ}\right)$ & $8.19 \pm 5.0$ & $7.3 \pm 4.3$ & 1.0470 .298 \\
\hline Post-mFTA $\left({ }^{\circ}\right)$ & $0.9 \pm 1.7$ & $0.3 \pm 1.8$ & $1.825 \quad 0.071$ \\
\hline FCRA $\left({ }^{\circ}\right)$ & $1.2 \pm 2.8$ & $0.7 \pm 2.0$ & $-0.906 \quad 0.361$ \\
\hline \multicolumn{4}{|l|}{ WOMAC } \\
\hline Pre-operation & \multicolumn{3}{|c|}{$103.69 \pm 17.97109 .06 \pm 13.50-1.6780 .097$} \\
\hline Postoperative 1 Month & $51.15 \pm 7.43$ & $48.72 \pm 6.31$ & $1.745 \quad 0.084$ \\
\hline Postoperative 3 Months & \multicolumn{3}{|c|}{$37.00 \pm 7.6038 .77 \pm 11.930-0.8740 .384$} \\
\hline Postoperative 12 Months & $30.90 \pm 4.94$ & $32.75 \pm 6.03$ & -1.7750 .097 \\
\hline \multicolumn{3}{|l|}{ level of satisfaction at 12 months after surgery } & 1.6680 .434 \\
\hline Very satisfied & 40 & 35 & \\
\hline Satisfied & 8 & 9 & \\
\hline Dissatisfied & 2 & 5 & \\
\hline
\end{tabular}

BMI: Body Mass Index, ASA: American Society of Anesthesiologists, Pre-mFTA: preoperative mechanical femorotibial angle; Post-mFTA, postoperative mechanical femorotibial angle; FCRA, femoral component rotation angle; CTMC, Cut Thickness of Medial Condyle; CTLC, Cut Thickness of Lateral Condyle.; PCA, Posterior Condylar axis.

Table 2 Comparison of pressure index for extension and flexion. 


\begin{tabular}{|c|c|c|c|c|c|}
\hline & & Medial compartment(pounds) & Lateral compartment(pounds) & $\mathrm{t}$ & P Value \\
\hline \multirow[t]{4}{*}{ GB } & Full extension & $18.5 \pm 4.4$ & $17.9 \pm 3.5$ & 0.813 & 0.420 \\
\hline & $45^{\circ}$ & $14.6 \pm 4.2$ & $13.6 \pm 2.714$ & 1.353 & 0.182 \\
\hline & $90^{\circ}$ & $11.3 \pm 5.1$ & $10.6 \pm 4.7$ & 0.645 & 0.522 \\
\hline & Full Flexion & $10.2 \pm 5.0$ & $9.1 \pm 4.9$ & 1.063 & 0.293 \\
\hline \multirow[t]{4}{*}{ MR } & Full extension & $17.5 \pm 5.1$ & $15.9 \pm 4.9$ & 1.594 & 0.117 \\
\hline & $45^{\circ}$ & $13.0 \pm 6.4$ & $11.1 \pm 5.2$ & 1.696 & 0.096 \\
\hline & $90^{\circ}$ & $11.4 \pm 7.1$ & $8.7 \pm 5.2$ & 2.772 & 0.008 \\
\hline & Full Flexion & $9.0 \pm 5.2$ & $7.1 \pm 4.7$ & 10.912 & 0.000 \\
\hline
\end{tabular}

Less than differential loading of 15 pounds between the medial and lateral compartments, was considered as adequately "balanced" according to previous studies.

Table 3 Comparison of gait analysis.

\begin{tabular}{rcccc}
\hline & GB & MR & t & P Value \\
\hline Spatiotemporal parameters & & & & \\
Walking speed (m/s) & $0.80 \pm 0.21$ & $0.75 \pm 0.28$ & 0.911 & 0.360 \\
\hline Step length (cm) & $51.00 \pm 3.46$ & $50.59 \pm 2.92$ & 0.474 & 0.639 \\
\hline Single support time (\%) & $39.61 \pm 6.80$ & $37.80 \pm 7.42$ & 1.185 & 0.239 \\
\hline Kinematic parameters & & & & \\
\hline Flexion angle $\left(^{\circ}\right)$ & $52.77 \pm 5.40$ & $49.52 \pm 7.80$ & 1.960 & 0.053 \\
\hline Extension angle $\left(^{\circ}\right)$ & $1.83 \pm 2.24$ & $2.10 \pm 2.38$ & -0.531 & 0.597 \\
\hline Sagittal max knee flexion $\left(^{\circ}\right)$ & $52.77 \pm 5.40$ & $49.52 \pm 7.83$ & 2.717 & $\mathbf{0 . 0 3 3}$ \\
\hline Coronal max knee flexion $\left({ }^{\circ}\right)$ & $10.16 \pm 2.67$ & $10.67 \pm 2.50$ & -0.896 & 0.629 \\
\hline Transversal knee rotation $\left({ }^{\circ}\right)$ & $3.60 \pm 2.51$ & $2.47 \pm 2.52$ & 2.077 & $\mathbf{0 . 0 4 1}$ \\
\hline
\end{tabular}

\section{Figures}




\section{CONSORT}

\section{TRANSPARENT REPORTING of TRIALS}

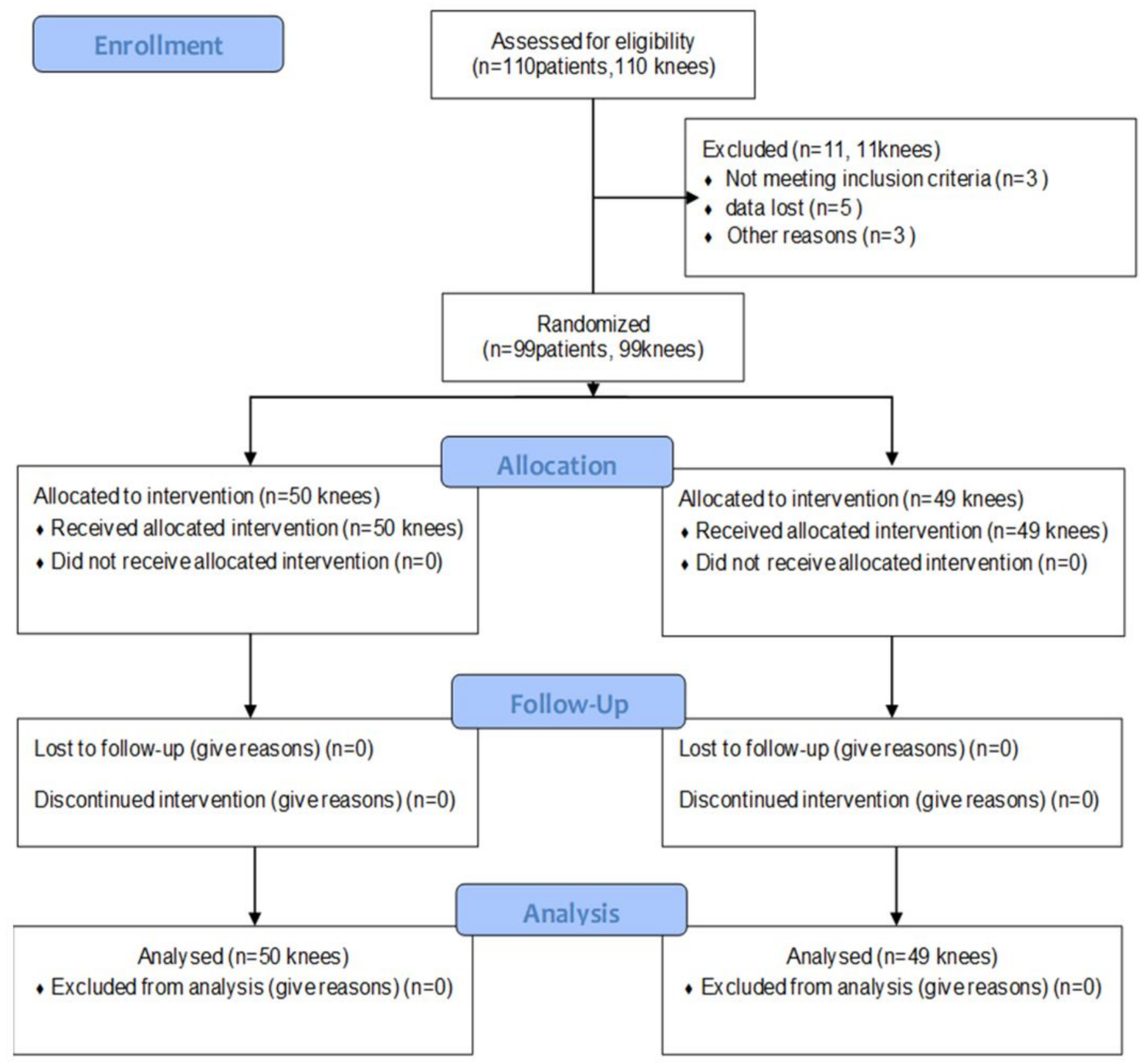

\section{Figure 1}

Flow diagram 


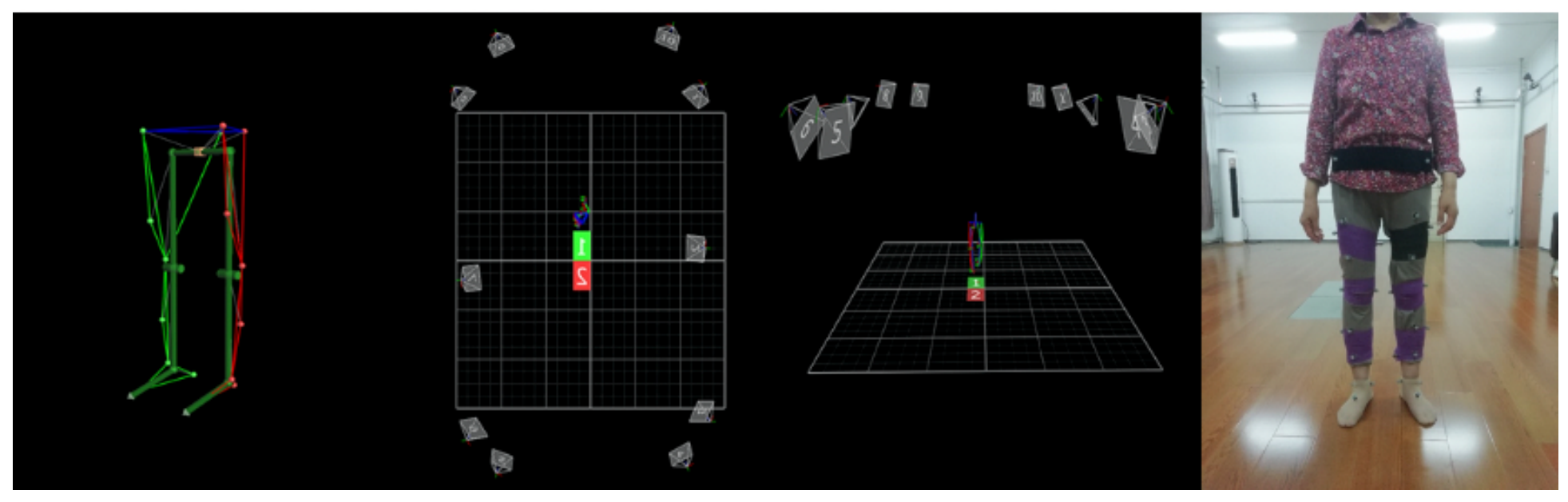

Figure 2

2a The Vicon 3D Motion Capture System (mainly including reflective markers, 10 capture cameras, and two 3D force stations). 2b Position of 26 reflective markers: anterior superior iliac spine and posterior superior iliac spine; Anterior and lateral thigh 1/3; The medial and lateral epicondyle of knee; Anterior and lateral calves 1/3; medial and lateral malleolus; Second metatarsal bone; Heel, at the same height as the second metatarsal bone.
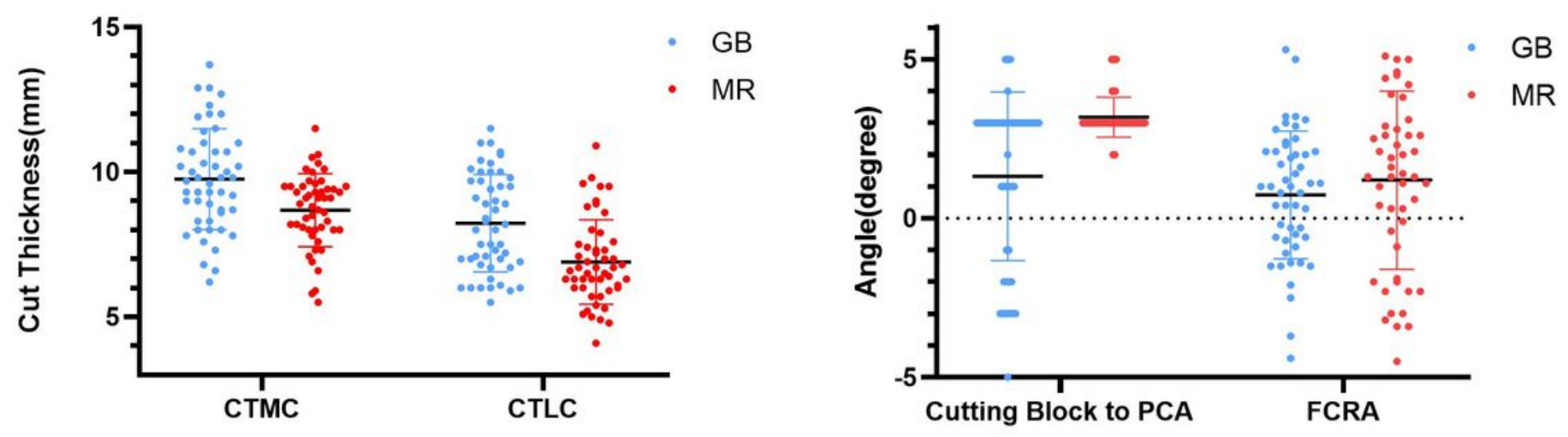

\section{Figure 3}

Cutting thickness of medial and lateral compartment. 


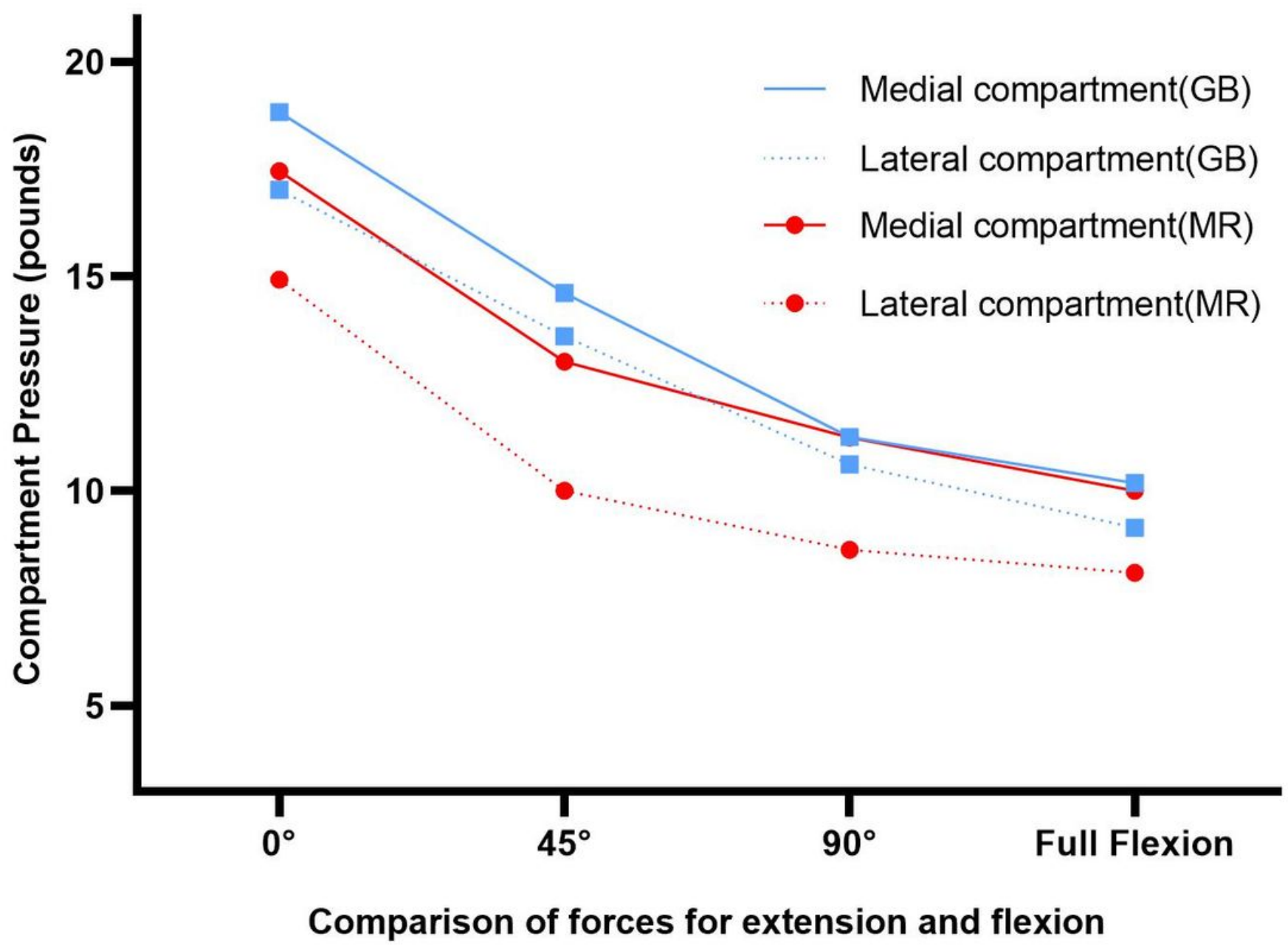

Figure 4

Pressure changes of medial and lateral compartment. 


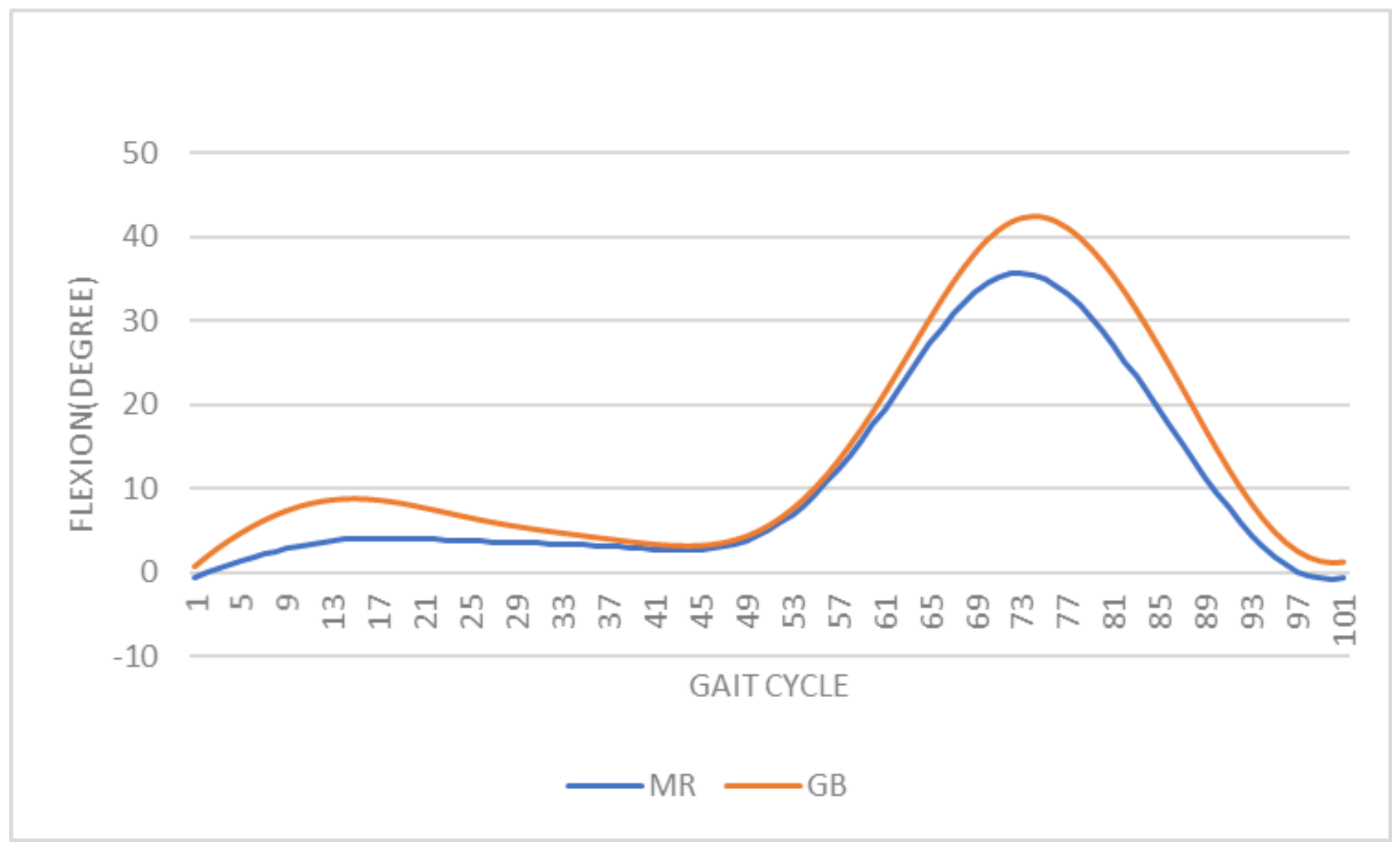

Figure 5

Sagittal knee flexion during gait cycle. 